\title{
Erratum
}

\section{Erratum: Gouws et al., On the Role of Suppression in Spatial Attention: Evidence from Negative BOLD in} Human Subcortical and Cortical Structures

In the article "On the Role of Suppression in Spatial Attention: Evidence from Negative BOLD in Human Subcortical and Cortical Structures" by André D. Gouws, Ivan Alvarez, David M. Watson, Maiko Uesaki, Jessica Rogers, and Antony B. Morland, which appeared on pages 10347-10360 of the July 30, 2014 issue, the fifth author last name was misspelled by the publisher. The corrected author line is: André D. Gouws, Ivan Alvarez, David M. Watson, Maiko Uesaki, Jessica Rodgers, and Antony B. Morland. This has been corrected on the online PDF version.

DOI:10.1523/JNEUROSCI.3727-14.2014 